\title{
Remarks on star-K-Menger spaces
}

\author{
Yan-Kui Song
}

\begin{abstract}
A space $X$ is star-K-Menger if for each sequence $\left(\mathcal{U}_{n}: n \in \mathbb{N}\right)$ of open covers of $X$ there exists a sequence $\left(K_{n}: n \in N\right)$ of compact subsets of $X$ such that $\left\{S t\left(K_{n}, \mathcal{U}_{n}\right): n \in \mathbb{N}\right\}$ is an open cover of $X$. In this paper, we construct an example of a Hausdorff star-Menger space that is not star-KMenger which gives an answer to a question of Song [12], and continue to investigate topological properties of star-K-Menger spaces.
\end{abstract}

\section{Introduction}

By a space, we mean a topological space. We give definitions of terms which are used in this paper. Let $\mathbb{N}$ denote the set of positive integers. Let $X$ be a space and $\mathcal{U}$ a collection of subsets of $X$. For $A \subseteq X$, let $S t(A, \mathcal{U})=\bigcup\{U \in \mathcal{U}: U \cap A \neq \varnothing\}$. As usual, we write $S t(x, \mathcal{U})$ instead of $S t(\{x\}, \mathcal{U})$.

Let $\mathcal{A}$ and $\mathcal{B}$ be collections of open covers of a space $X$. Then the symbol $S_{1}(\mathcal{A}, \mathcal{B})$ denotes the selection hypothesis that for each sequence $\left(\mathcal{U}_{n}: n \in \mathbb{N}\right)$ of elements of $\mathcal{A}$ there exists a sequence $\left(U_{n}: n \in \mathbb{N}\right)$ such that for each $n \in \mathbb{N}$, $U_{n} \in \mathcal{U}_{n}$ and $\left\{U_{n}: n \in \mathbb{N}\right\}$ is an element of $\mathcal{B}$. The symbol $S_{\text {fin }}(\mathcal{A}, \mathcal{B})$ denotes the selection hypothesis that for each sequence $\left(\mathcal{U}_{n}: n \in \mathbb{N}\right)$ of elements of $\mathcal{A}$ there exists a sequence $\left(\mathcal{V}_{n}: n \in \mathbb{N}\right)$ such that for each $n \in \mathbb{N}, \mathcal{V}_{n}$ is a finite subset of $\mathcal{U}_{n}$ and $\bigcup_{n \in \mathbb{N}} \mathcal{V}_{n}$ is an element of $\mathcal{B}$ (see $[6,11]$ ).

Kočinac $[7,8]$ introduced star selection hypothesis similar to the previous ones. Let $\mathcal{A}$ and $\mathcal{B}$ be collections of open covers of a space $X$. Then:

(A) The symbol $S_{\text {fin }}^{*}(\mathcal{A}, \mathcal{B})$ denotes the selection hypothesis that for each sequence $\left(\mathcal{U}_{n}: n \in \mathbb{N}\right)$ of elements of $\mathcal{A}$ there exists a sequence $\left(\mathcal{V}_{n}: n \in \mathbb{N}\right)$ such

Received by the editors in September 2014 - In revised form in June 2015.

Communicated by E. Colebunders.

2010 Mathematics Subject Classification : 54D20, 54C10.

Key words and phrases : Selection principles, star-Menger, strongly star-Menger, star-KMenger, starcompact, star Lindelöf, strongly starcompact, strongly star Lindelöf, star-L-Lindelöf. 
that for each $n \in \mathbb{N}, \mathcal{V}_{n}$ is a finite subset of $\mathcal{U}_{n}$ and $\bigcup_{n \in \mathbb{N}}\left\{\operatorname{St}\left(V, \mathcal{U}_{n}\right): V \in \mathcal{V}_{n}\right\}$ is an element of $\mathcal{B}$.

(B) The symbol $S S_{\text {comp }}^{*}(\mathcal{A}, \mathcal{B})\left(S S_{\text {fin }}^{*}(\mathcal{A}, \mathcal{B})\right)$ denotes the selection hypothesis that for each sequence $\left(\mathcal{U}_{n}: n \in \mathbb{N}\right)$ of elements of $\mathcal{A}$ there exists a sequence $\left(K_{n}: n \in N\right)$ of compact (resp., finite) subsets of $X$ such that $\left\{\operatorname{St}\left(K_{n}, \mathcal{U}_{n}\right)\right.$ : $n \in \mathbb{N}\} \in \mathcal{B}$.

Let $\mathcal{O}$ denote the collection of all open covers of $X$.

Definition 1.1. $([7,8])$ A space $X$ is said to be star-Menger if it satisfies the selection hypothesis $S_{\text {fin }}^{*}(\mathcal{O}, \mathcal{O})$.

Definition 1.2. ([7,8,12]) A space $X$ is said to be star-K-Menger (strongly star-Men$g e r)$ if it satisfies the selection hypothesis $S S_{\text {comp }}^{*}(\mathcal{O}, \mathcal{O})\left(\right.$ resp., $\left.S S_{\text {fin }}^{*}(\mathcal{O}, \mathcal{O})\right)$.

Definition 1.3. ([3,9]) A space $X$ is said to be K-starcompact if for every open cover $\mathcal{U}$ of $X$ there exists a compact subset $F$ of $X$ such that $S t(F, \mathcal{U})=X$.

From the definitions, it is clear that every K-starcompact space is star-K-Menger, every strongly star-Menger space is star-K-Menger and every star-K-Menger space is star-Menger.

The author [12] studied the star-K-Menger and related spaces, and investigated topological properties of star-K-Menger spaces. The author [12] constructed a $T_{1}$ star-Menger space which is not star-K-Menger and asked if there exists Hausdorff or Tychonoff example. The purpose of this paper is to show the statement stated in the abstract, which gives an answer to the question above of Song in class of Hausdorff spaces, and continue to investigate topological properties of star-K-Menger spaces.

Throughout this paper, let $\omega$ denote the first infinite cardinal, $\omega_{1}$ the first uncountable cardinal, $\mathfrak{c}$ the cardinality of the set of all real numbers. For a cardinal $\kappa$, let $\kappa^{+}$be the smallest cardinal greater than $\kappa$. For each pair of ordinals $\alpha, \beta$ with $\alpha<\beta$, we write $[\alpha, \beta)=\{\gamma: \alpha \leq \gamma<\beta\},(\alpha, \beta]=\{\gamma: \alpha<\gamma \leq \beta\}$, $(\alpha, \beta)=\{\gamma: \alpha<\gamma<\beta\}$ and $[\alpha, \beta]=\{\gamma: \alpha \leq \gamma \leq \beta\}$. As usual, a cardinal is an initial ordinal and an ordinal is the set of smaller ordinals. A cardinal is often viewed as a space with the usual order topology. Other terms and symbols that we do not define follow [5].

\section{Star-K-Menger spaces and related spaces}

In this section, first we construct the example stated in the abstract.

Lemma 2.1. If $X=\bigcup\left\{X_{n}: n \in \mathbb{N}\right\}$ and each $X_{n}$ is star-Menger, then $X$ is starMenger.

Proof. Let $\left(\mathcal{U}_{n}: n \in \mathbb{N}\right)$ be a sequence of open covers of $X$ and let $\mathbb{N}=\mathbb{N}_{1} \cup \mathbb{N}_{2} \cup$ .. be a partition of $\mathbb{N}$ into infinitely many infinite pairwise disjoint subsets. For each $n \in \mathbb{N}$, since $X_{n}$ is star-Menger, thus for the sequence $\left(\mathcal{U}_{m}: m \in \mathbb{N}_{n}\right)$ of open 
covers of $X$, there exists a sequence $\left\{\mathcal{V}_{m}: m \in \mathbb{N}_{n}\right\}$ such that for each $m \in \mathbb{N}_{n}$, $\mathcal{V}_{m}$ is a finite subset of $\mathcal{U}_{m}$ and

$$
X_{n} \subseteq \bigcup_{m \in \mathbb{N}_{n}} S t\left(\cup \mathcal{V}_{m}, \mathcal{U}_{m}\right)
$$

Hence the sequence $\left\{\mathcal{V}_{n}: n \in \mathbb{N}\right\}$ witnesses for $\left\{\mathcal{U}_{n}: n \in \mathbb{N}\right\}$ that $X$ is starMenger. Therefore we complete the proof.

It is clear that every compact space is star-Menger. Thus we have the following corollary by Lemma 2.1.

Corollary 2.2. If $X$ is a $\sigma$-compact space, then $X$ is star-Menger.

Example 2.3. There exists a Hausdorff star-Menger space which is not star-K-Menger.

Proof. Let

$$
\begin{aligned}
& A=\left\{a_{\alpha}: \alpha<\mathfrak{c}\right\}, B=\left\{b_{n}: n \in \omega\right\} \\
& \text { and } Y=\left\{\left\langle a_{\alpha}, b_{n}\right\rangle: \alpha<\mathfrak{c}, n \in \omega\right\}
\end{aligned}
$$

and let

$$
X=Y \cup A \cup\{a\} \text { where } a \notin Y \cup A .
$$

We topologize $X$ as follows: every point of $Y$ is isolated; a basic neighborhood of a point $a_{\alpha} \in A$ for each $\alpha<\mathfrak{c}$ takes the form

$$
U_{a_{\alpha}}(n)=\left\{a_{\alpha}\right\} \cup\left\{\left\langle a_{\alpha}, b_{m}\right\rangle: m>n\right\} \text { for } n \in \omega
$$

and a basic neighborhood of a point $a$ takes the form

$$
U_{a}(F)=\{a\} \cup \cup\left\{\left\langle a_{\alpha}, b_{n}\right\rangle: a_{\alpha} \in A \backslash F, n \in \omega\right\} \text { for a countable subset } F \text { of } A .
$$

Clearly, $X$ is a Hausdorff space by the construction of the topology of $X$. However, $X$ is not regular, since the point $a$ can not be separated from the closed subset $A$ by disjoint open subsets of $X$.

Now we show that $X$ is star-Menger. To this end, let $\left(\mathcal{U}_{n}: n \in \mathbb{N}\right)$ be a sequence of open covers of $X$. Without loss of generality, we assume that $\mathcal{U}_{n}$ consists of basic open sets of $X$ for each $n \in \mathbb{N}$. Since $\mathcal{U}_{1}$ is an open cover of $X$, there exists $U_{a} \in \mathcal{U}_{1}$ such that $a \in U_{a}$. By assumption, there exists a countable subset $F$ of $A$ such that

$$
U_{a}=U_{a}(F)=\{a\} \cup \cup\left\{\left\langle a_{\alpha}, b_{n}\right\rangle: a_{\alpha} \in A \backslash F, n \in \omega\right\}
$$

by the definition of the topology of $X$, thus we have

$$
F \cup U_{a} \subseteq S t\left(U_{a}, \mathcal{U}\right)
$$

For each $a_{\alpha} \in F$, let

$$
B_{a_{\alpha}}=\left\{a_{\alpha}\right\} \cup\left\{\left\langle a_{\alpha}, b_{n}\right\rangle: n \in \omega\right\} .
$$

Then $B_{a_{\alpha}}$ is a compact subset of $X$ by the definition of the topology of $X$. Let $A=\cup_{a_{\alpha} \in F} B_{a_{\alpha}}$. Then $A$ is $\sigma$-compact and $X=A \cup F \cup U_{a}$. Thus $A$ is star-Menger 
by Corollary 2.2. For the sequence $\left(\mathcal{U}_{n}: n>1\right)$ of open covers of $X$, there exists a sequence $\left(\mathcal{V}_{n}: n>1\right)$ such that for each $n>1, \mathcal{V}_{n}$ is a finite subset of $\mathcal{U}_{n}$ and

$$
A \subseteq \bigcup_{n>1} S t\left(\cup \mathcal{V}_{n}, \mathcal{U}_{n}\right)
$$

Let $\mathcal{V}_{1}=\left\{U_{a}\right\}$. Then the sequence $\left\{\mathcal{V}_{n}: n \in \mathbb{N}\right\}$ witnesses for $\left\{\mathcal{U}_{n}: n \in \mathbb{N}\right\}$ that $X$ is star-Menger.

Next we show that $X$ is not star-K-Menger. For each $\alpha<\mathfrak{c}$, let

$$
U_{\alpha}=\left\{a_{\alpha}\right\} \cup\left\{\left\langle a_{\alpha}, b_{n}\right\rangle: n \in \omega\right\} \text { and } U=U_{a}(\varnothing) .
$$

Then $U_{\alpha}$ is open and closed in $X$ by the construction of the topology of $X$ and

$$
U_{\alpha} \cap U_{\alpha^{\prime}}=\varnothing \text { for } \alpha \neq \alpha^{\prime}
$$

For $n \in \mathbb{N}$, let

$$
\mathcal{U}_{n}=\left\{U_{\alpha}: \alpha<\mathfrak{c}\right\} \cup\{U\} .
$$

Let us consider the sequence $\left(\mathcal{U}_{n}: n \in \mathbb{N}\right)$ of open covers of $X$. We only show that $\bigcup_{n \in \mathbb{N}} s t\left(K_{n}, \mathcal{U}_{n}\right) \neq X$ for any sequence $\left(K_{n}: n \in \mathbb{N}\right)$ of compact subsets of $X$. Let $\left(K_{n}: n \in \mathbb{N}\right)$ be any sequence of compact subsets of $X$. For each $n \in \mathbb{N}$, since $K_{n}$ is compact, then there exists $\alpha_{n}<\mathfrak{c}$ such that $K_{n} \cap U_{\alpha}=\varnothing$ for each $\alpha>\alpha_{n}$. Let $\alpha^{\prime}=\sup \left\{\alpha_{n}: n \in \mathbb{N}\right\}$. If we pick $\beta>\alpha^{\prime}$. Then $U_{\beta} \cap K_{n}=\varnothing$ for each $n \in \mathbb{N}$. Hence $a_{\beta} \notin S t\left(K_{n}, \mathcal{U}_{n}\right)$ for each $n \in \mathbb{N}$, since $U_{\beta}$ is the only element of $\mathcal{U}_{n}$ containing the point $a_{\beta}$ for each $n \in \mathbb{N}$, which shows that $X$ is not star-K-Menger. Thus we complete the proof.

Question 2.1. The author does not know if there exists a regular (or Tychonoff) starMenger space which is not star-K-Menger.

The author [12] showed that a regular-closed subset of a Tychonoff star-KMenger space $X$ need not be star-K-Menger. Next we construct an example showing that assuming $\mathfrak{d}=\mathfrak{c}$, there exists a Tychonoff star-K-Menger space having a regular-closed $G_{\delta}$-subspace which is not star-K-Menger by using the following example from $[1,10]$. We make use of two of the cardinals defined in [3,4]. Define ${ }^{\omega} \omega$ as the set of all functions from $\omega$ to itself. For all $f, g \in \in^{\omega} \omega$, we say $f \leq^{*} g$ if and only if $f(n) \leq g(n)$ for all but finitely many $n$. The unbounding number, denoted by $\mathfrak{b}$, is the smallest cardinality of an unbounded subset of $\left(\omega \omega, \leq^{*}\right)$. The dominating number, denoted by $\mathfrak{d}$, is the smallest cardinality of a cofinal subset of $\left({ }^{\omega} \omega, \leq^{*}\right)$. It is not difficult to show that $\omega_{1} \leq \mathfrak{b} \leq \mathfrak{d} \leq \mathfrak{c}$ and it is known that $\omega_{1}<\mathfrak{b}=\mathfrak{c}, \omega_{1}<\mathfrak{d}=\mathfrak{c}$ and $\omega_{1} \leq \mathfrak{b}<\mathfrak{d}=\mathfrak{c}$ are all consistent with the axioms of ZFC (see [3] for details).

Example 2.4. ([1,9]) Let $\mathcal{A}$ be an almost disjoint family of infinite subsets of $\omega$ (i.e., the intersection of every two distinct elements of $\mathcal{A}$ is finite) and Let $X=\omega \cup \mathcal{A}$ be the Isbell-Mrówka space constructed from $\mathcal{A}([4,5])$. Then $X$ is strongly star-Menger if and only if $|\mathcal{A}|<\mathfrak{d}$.

For the next example, we need the following Lemma. For a Tychonoff space $X$, let $\beta X$ denote the Cech-Stone compactification of $X$. 
Lemma 2.5. Let $\kappa$ be infinite cardinal and $D=\left\{d_{\alpha}: \alpha<\kappa\right\}$ be a discrete space of cardinality $\kappa$. Then the subspace $X=(\beta D \times \kappa) \cup(D \times\{\kappa\})$ of the product space $\beta D \times(\kappa+1)$ is not star-K-Menger.

Proof. We show that $X$ is not star-K-Menger. For each $\alpha<\kappa$, let $U_{\alpha}=\left\{d_{\alpha}\right\} \times$ $(\alpha, \kappa]$. Then $U_{\alpha}$ is open in $X$ and

$$
U_{\alpha} \cap U_{\alpha^{\prime}}=\varnothing \text { for each } \alpha \neq \alpha^{\prime}
$$

For each $n \in \mathbb{N}$, let

$$
\mathcal{U}_{n}=\left\{U_{\alpha}: \alpha<\kappa\right\} \cup\{\beta D \times \kappa\} .
$$

Then $\mathcal{U}_{n}$ is an open cover of $X$. Let us consider the sequence $\left(\mathcal{U}_{n}: n \in \mathbb{N}\right)$ of open covers of $X$. It suffices to show that $\bigcup_{n \in \mathbb{N}} S t\left(K_{n}, \mathcal{U}_{n}\right) \neq X$ for any sequence $\left(K_{n}: n \in \mathbb{N}\right)$ of compact subsets of $X$. Let $\left(K_{n}: n \in \mathbb{N}\right)$ be any sequence of compact subsets of $X$. For each $n \in \mathbb{N}$, since $K_{n}$ is compact and $\left\{\left\langle d_{\alpha}, \kappa\right\rangle: \alpha<\kappa\right\}$ is a discrete closed subset of $X$, the set $K_{n} \cap\left\{\left\langle d_{\alpha}, \omega\right\rangle: \alpha<\mathfrak{c}\right\}$ is finite. Then there exists $\alpha_{n}<\kappa$ such that

$$
K_{n} \cap\left\{\left\langle d_{\alpha}, \kappa\right\rangle: \alpha>\alpha_{n}\right\}=\varnothing .
$$

Let $\alpha^{\prime}=\sup \left\{\alpha_{n}: n \in \mathbb{N}\right\}$. Then $\alpha^{\prime}<\kappa$ and

$$
\left(\bigcup_{n \in \mathbb{N}} K_{n}\right) \cap\left\{\left\langle d_{\alpha}, \kappa\right\rangle: \alpha>\alpha^{\prime}\right\}=\varnothing .
$$

On the other hand, for each $n \in \mathbb{N}$, let $A_{n}=\left\{\alpha:\left\langle d_{\alpha}, \kappa\right\rangle \in K_{n}\right\}$. Then $A_{n}$ is finite, since $K_{n}$ is compact and $\left\{\left\langle d_{\alpha}, \kappa\right\rangle: \alpha<\kappa\right\}$ is discrete and closed in $X$. Let $K_{n}^{\prime}=K_{n} \backslash \bigcup\left\{U_{\alpha}: \alpha \in A_{n}\right\}$. Then $K_{n}^{\prime}$ is closed in $K_{n}$ and $K_{n}^{\prime} \subseteq \beta D \times \kappa$. Hence $\pi\left(K_{n}^{\prime}\right)$ is a compact subset of the countably compact space $\kappa$, where $\pi: \beta D \times \kappa \rightarrow$ $\kappa$ is the projection, thus there exists $\alpha_{n}^{\prime}<\kappa$ such that $\pi\left(K_{n}^{\prime}\right) \cap\left(\alpha_{n}^{\prime}, \kappa\right)=\varnothing$. Let $\alpha^{\prime \prime}=\sup \left\{\alpha_{n}^{\prime}: n \in \mathbb{N}\right\}$. Then $\alpha^{\prime \prime}<\kappa$. If we pick $\beta>\max \left\{\alpha^{\prime}, \alpha^{\prime \prime}\right\}$. Then $U_{\beta} \cap K_{n}=\varnothing$ for each $n \in \mathbb{N}$. Hence $\left\langle d_{\beta}, \kappa\right\rangle \notin S t\left(K_{n}, \mathcal{U}_{n}\right)$ for each $n \in \mathbb{N}$, since $U_{\beta}$ is the only element of $\mathcal{U}_{n}$ containing the point $\left\langle d_{\beta}, \kappa\right\rangle$ for each $n \in \mathbb{N}$, which shows that $X$ is not star-K-Menger.

Recall from $[3,9]$ that a space $X$ is said to be strongly starcompact if for every open cover $\mathcal{U}$ of $X$ there exists a finite subset $F$ of $X$ such that $S t(F, \mathcal{U})=X$. It is well known that strongly starcompactness is equivalent to countably compactness for Hausdorff spaces (see [3,9]).

Example 2.6. Assuming $\mathfrak{d}=\mathfrak{c}$, there exists a Tychonoff star-K-Menger space having $a$ regular-closed $G_{\delta}$-subspace which is not star-K-Menger.

Proof. Assuming $\mathfrak{d}=\mathfrak{c}$, let $S_{1}=\omega \cup \mathcal{A}$ be the same space $X$ as in the construction of Example 2.4 with $|\mathcal{A}|=\omega_{1}$. Then $S_{1}$ is strongly star-Menger by Example 2.4. Hence $S_{1}$ is star-K-Menger, since every strongly star-Menger space is star-KMenger.

Let $D=\left\{d_{\alpha}: \alpha<\omega_{1}\right\}$ be a discrete space of cardinality $\omega_{1}$ and let

$$
S_{2}=\left(\beta D \times \omega_{1}\right) \cup\left(D \times\left\{\omega_{1}\right\}\right)
$$


be the subspace of the product space $\beta D \times\left(\omega_{1}+1\right)$. Then $S_{2}$ is not star-K-Menger by Lemma 2.5 .

Let $\pi: \mathcal{A} \rightarrow D \times\left\{\omega_{1}\right\}$ be a bijection and let $X$ be the quotient image of the disjoint sum $S_{1} \oplus S_{2}$ by identifying $A$ of $S_{1}$ with $\pi(A)$ of $S_{2}$ for every $A \in \mathcal{A}$. Let $\varphi: S_{1} \oplus S_{2} \rightarrow X$ be the quotient map. Then $\varphi\left(S_{2}\right)$ is a regular-closed subspace of $X$. For each $n \in \omega$, let $F_{n}=\{m \in \omega: m \leq n\}$. For each $n \in \omega$, let

$$
U_{n}=\varphi\left(\left\{\{A\} \cup\left(A \backslash F_{n}\right): A \in \mathcal{A}\right\}\right) \cup \varphi\left(\beta D \times \omega_{1}\right) .
$$

Then $U_{n}$ is open in $X$ and $\varphi\left(S_{2}\right)=\bigcap_{n \in \omega} U_{n}$. Thus $\varphi\left(S_{2}\right)$ is a regular-closed $G_{\delta}$-subspace of $X$. However $\varphi\left(S_{2}\right)$ is not star-K-Menger, since it is homeomorphic to $S_{2}$.

Finally we show that $X$ is star-K-Menger. To this end, let $\left\{\mathcal{U}_{n}: n \in \mathbb{N}\right\}$ be a sequence of open covers of $X$. Since $\varphi\left(\beta D \times \omega_{1}\right)$ is homeomorphic to $\beta D \times \omega_{1}$, and consequently $\varphi\left(\beta D \times \omega_{1}\right)$ is countably compact, we can find a finite subset $K_{1}$ of $\varphi\left(\beta D \times \omega_{1}\right)$ such that

$$
\varphi\left(\beta D \times \omega_{1}\right) \subseteq S t\left(K_{1}, \mathcal{U}_{1}\right)
$$

On the other hand, Since $\varphi\left(S_{1}\right)$ is homeomorphic to $S_{1}$ and consequently $\varphi\left(S_{1}\right)$ is star-K-Menger. Thus for the sequence $\left(\mathcal{U}_{n}: n>1\right)$ of open covers of $X$, there exists a sequence $\left\{K_{n}: n>1\right\}$ of compact subsets of $\varphi\left(S_{1}\right)$ such that

$$
\varphi\left(S_{1}\right) \subseteq \bigcup_{n>1} S t\left(K_{n}, \mathcal{U}_{n}\right)
$$

Hence the sequence $\left\{K_{n}: n \in \mathbb{N}\right\}$ of compact subsets of $X$ witnesses for $\left\{\mathcal{U}_{n}: n \in \mathbb{N}\right\}$ that $X$ is star-K-Menger. Therefore we complete the proof.

Question 2.2. The author does not know if there exists an example in ZFC showing that a regular-closed $G_{\delta}$-subspace of a star-K-Menger space is not star-K-Menger.

The following example gives an answer to Question 2.2 for Hausdorff spaces.

Example 2.7. There exists a Hausdorff star-K-Menger space having a regular-closed $\mathrm{G}_{\delta}$-subspace which is not star-K-Menger.

Proof. Let $D=\left\{d_{\alpha}: \alpha<\mathfrak{c}\right\}$ be a discrete space of cardinality $\mathfrak{c}$ and let

$$
S_{1}=(\beta D \times(\mathfrak{c}+1)) \cup(D \times\{\mathfrak{c}\})
$$

be the subspace of the product space $\beta D \times(\mathfrak{c}+1)$. Then $S_{2}$ is not star-K-Menger by Lemma 2.5 .

Let $S_{2}=Y \cup A \cup\{a\}$ where $a \notin Y \cup A$ be the same space $X$ as the proof of Example 2.3. We topologize $S_{2}$ as follows: every point of $Y \cup A$ has the same neighborhood as the proof of Example 2.3 and a basic neighborhood of a point $a$ takes the form

$$
U_{a}(F)=\{a\} \cup \cup\left\{\left\langle a_{\alpha}, b_{n}\right\rangle: a_{\alpha} \in A \backslash F, n \in \omega\right\} \text { for a finite subset } F \text { of } A .
$$

Clearly, $S_{2}$ is a Hausdorff space, but is not regular. 
Now we show that $S_{2}$ is star-K-Menger. We only show that $S_{2}$ is K-starcompact, since every K-starcompact space is star-K-Menger. To this end, let $\mathcal{U}$ be an open cover of $S_{2}$. Without loss of generality, we assume that $\mathcal{U}$ consists of basic open sets of $S_{2}$. Since $\mathcal{U}$ is an open cover of $S_{2}$, there exists $U_{a} \in \mathcal{U}$ such that $a \in \mathcal{U}_{a}$. By assumption, there exists a finite subset $F$ of $A$ such that

$$
U_{a}=U_{a}(F)=\{a\} \cup \cup\left\{\left\langle a_{\alpha}, b_{n}\right\rangle: a_{\alpha} \in A \backslash F, n \in \omega\right\}
$$

by the definition of the topology of $S_{2}$, thus we have

$$
U_{a} \subseteq S t(a, \mathcal{U})
$$

For each $a_{\alpha} \in F$, let

$$
B_{a_{\alpha}}=\left\{a_{\alpha}\right\} \cup\left\{\left\langle a_{\alpha}, b_{n}\right\rangle: n \in \omega\right\} .
$$

Then $B_{a_{\alpha}}$ is a compact subset of $S_{2}$ by the definition of the topology of $S_{2}$. On the other hand, for each $a_{\alpha} \in A \backslash F$, there exists $U_{a_{\alpha}} \in \mathcal{U}$ such that $a_{\alpha} \in U_{a_{\alpha}}$. Thus there exists $\alpha_{n} \in \omega$ such that $\left\langle a_{\alpha}, \alpha_{n}\right\rangle \in U_{a_{\alpha}}$. Let $C=\left\{\left\langle a_{\alpha}, \alpha_{n}\right\rangle: a_{\alpha} \in A \backslash F\right\}$. Then $C \cup\{a\}$ is a compact subset of $S_{2}$ by the definition of $S_{2}$ and

$$
A \backslash F \subseteq S t(C \cup\{a\}, \mathcal{U})
$$

Set

$$
K=\{a\} \cup C \cup\left\{B_{a_{\alpha}}: a_{\alpha} \in F\right\} .
$$

Then $K$ is a compact subset of $S_{2}$ such that $S_{2}=S t(K, \mathcal{U})$, which shows that $S_{2}$ is K-starcompact.

We assume $S_{1} \cap S_{2}=\varnothing$. Let $\pi: D \times\{\mathfrak{c}\} \rightarrow A$ be a bijection and let $X$ be the quotient image of the disjoint sum $S_{1} \oplus S_{2}$ by identifying $\left\langle d_{\alpha}, \mathfrak{c}\right\rangle$ of $S_{1}$ with $\pi\left(\left\langle d_{\alpha}, \mathfrak{c}\right\rangle\right)$ of $S_{2}$ for every $\alpha<\mathfrak{c}$. Let $\varphi: S_{1} \oplus S_{2} \rightarrow X$ be the quotient map. Clearly, $\varphi\left(S_{1}\right)$ is a regular-close subspace of $X$. For each $n \in \omega$, let

$$
U_{n}=\pi\left(S_{1} \cup\left\{\left\langle a_{\alpha}, b_{m}\right\rangle: m>n, \alpha<\mathfrak{c}\right\}\right) .
$$

Then $U_{n}$ is open in $X$ and $\varphi\left(S_{1}\right)=\bigcap_{n \in \omega} U_{n}$. Thus $\varphi\left(S_{1}\right)$ is a regular-closed $G_{\delta}$-subspace of $X$. However $\varphi\left(S_{1}\right)$ is not star-K-Menger, since it is homeomorphic to $S_{1}$.

Similarly to the proof that the space $X$ in Example 2.6 is star-K-Menger, we can prove that $X$ is star-K-Menger. Thus we complete the proof.

We give a positive result on star-K-Menger spaces:

Theorem 2.8. An open and closed subset of a star-K-Menger space is star-K-Menger.

Proof. Let $X$ be a star-K-Menger space and let $Y$ be an open and closed subset of $X$. To show that $Y$ is star-K-Menger. Let $\left(\mathcal{U}_{n}: n \in \mathbb{N}\right)$ be a sequence of open covers of $Y$, we have to find a sequence $\left\{F_{n}: n \in \mathbb{N}\right\}$ of compact subsets of $Y$ such that

$$
Y=\bigcup_{n \in \mathbb{N}} S t\left(F_{n}, \mathcal{U}_{n}\right)
$$

For each $n \in \mathbb{N}$, let

$$
\mathcal{V}_{n}=\mathcal{U}_{n} \cup\{X \backslash Y\}
$$


Then $\left\{\mathcal{V}_{n}: n \in \mathbb{N}\right\}$ is a sequence of open covers of $X$, there exists a sequence $\left\{F_{n}^{\prime}: n \in \mathbb{N}\right\}$ of compact subsets of $X$ such that $X=\bigcup_{n \in \mathbb{N}} S t\left(F_{n}^{\prime}, \mathcal{V}_{n}\right)$, since $X$ is star-K-Menger. For each $n \in \mathbb{N}$, let $F_{n}=F_{n}^{\prime} \cap Y$. Thus $\left\{F_{n}: n \in \mathbb{N}\right\}$ is a sequence of compact subsets of $Y$, since $Y$ is a closed subset of $X$. For each $y \in Y$, if $y \in S t\left(F_{n}^{\prime}, \mathcal{V}_{n}\right)$, then $y \in S t\left(F_{n}, \mathcal{U}_{n}\right)$ by the construction of $\mathcal{V}_{n}$. Hence the sequence $\left\{F_{n}: n \in \mathbb{N}\right\}$ of compact subsets of $Y$ witnesses for $\left\{\mathcal{U}_{n}: n \in \mathbb{N}\right\}$ that $Y$ is star-K-Menger. Therefore we complete the proof.

For a space $X$, recall that the Alexandroff duplicate $A(X)$ of a space $X$, denoted by $A(X)$, is constructed in the following way: The underlying set $A(X)$ is $X \times\{0,1\}$; each point of $X \times\{1\}$ is isolated and a basic neighborhood of $\langle x, 0\rangle \in X \times\{0\}$ is a set of the form $(U \times\{0\}) \cup((U \times\{1\}) \backslash\{\langle x, 1\rangle\})$, where $U$ is a neighborhood of $x$ in $X$. It is well known that a space $X$ is compact if and only if so is $A(X)$. In the following, we give two examples to show that the result can not be generalized to star-K-Menger. For a Tychonoff space $X$, let $\beta X$ denote the Čech-Stone compactification of $X$. We give a lemma from [9]. Here we give the proof for the sake of completeness.

Lemma 2.9. Let $\kappa$ be infinite cardinal and $D=\left\{d_{\alpha}: \alpha<\kappa\right\}$ be a discrete space of cardinality $\kappa$. Then the subspace $X=\left(\beta D \times \kappa^{+}\right) \cup\left(D \times\left\{\kappa^{+}\right\}\right)$of the product space $\beta D \times\left(\kappa^{+}+1\right)$ is K-starcompact (hence star-K-Menger).

Proof. We only show that $X$ is K-starcompact. To this end, let $\mathcal{U}$ be an open cover of $X$. For each $\alpha<\kappa$, there exist $\beta_{\alpha}<\kappa^{+}$and $U_{\alpha} \in \mathcal{U}$ such that $\left\{d_{\alpha}\right\} \times\left(\beta_{\alpha}, \mathcal{K}^{+}\right] \subseteq$ $U_{\alpha}$. Let $\beta=\sup \left\{\beta_{\alpha}: \alpha<\kappa\right\}$. Then $\beta<\kappa^{+}$. Let $K_{1}=\beta D \times\{\beta+1\}$. Then $K_{1}$ is a compact subset of $X$ and $D \times\left\{\mathcal{K}^{+}\right\} \subseteq S t\left(K_{1}, \mathcal{U}\right)$. On the other hand, since $\beta D \times\left[0, \kappa^{+}\right)$is countably compact, there exists a finite subset $K_{2}$ of $\beta D \times\left[0, \kappa^{+}\right)$ such that $\beta D \times\left[0, \kappa^{+}\right) \subseteq S t\left(K_{2}, \mathcal{U}\right)$. If we put $K=K_{1} \cup K_{2}$, then $K$ is a compact subset of $X$ such that $X=S t(K, \mathcal{U})$, which shows that $X$ is K-starcompact.

Example 2.10. There exists a Tychonoff star-K-Menger space $X$ such that $A(X)$ is not star-K-Menger.

Proof. Let $D=\left\{d_{\alpha}: \alpha<\mathfrak{c}\right\}$ be a discrete space of the cardinality $\mathfrak{c}$, and let

$$
X=\left(\beta D \times \mathfrak{c}^{+}\right) \cup\left(D \times\left\{\mathfrak{c}^{+}\right\}\right)
$$

be the subspace of the product space $\beta D \times\left(\mathfrak{c}^{+}+1\right)$. Then $X$ is star-K-Menger by Lemma 2.9.

However $A(X)$ is not star-K-Menger. In fact, since $D \times\left\{\mathfrak{c}^{+}\right\}$is a discrete closed subset of $X$ with $\left|D \times\left\{\mathfrak{c}^{+}\right\}\right|=\mathfrak{c}$, then the set $\left(D \times\left\{\mathfrak{c}^{+}\right\}\right) \times\{1\}$ is an open and closed subset of $A(X)$ with $\left|\left(D \times\left\{\mathfrak{c}^{+}\right\}\right) \times\{1\}\right|=\mathfrak{c}$, and each point $\left\langle\left\langle d_{\alpha}, \mathfrak{c}^{+}\right\rangle, 1\right\rangle$ is isolated for each $\alpha<\mathfrak{c}$. Hence $A(X)$ is not star-K-Menger by Theorem 2.8, since $\left(D \times\left\{\mathfrak{c}^{+}\right\}\right) \times\{1\}$ is not star-K-Menger.

Now we give a positive result. For showing the result, we need a lemma from [2]. For a space $X$, the extent $e(X)$ of $X$ is the supremum of cardinalities of closed discrete subsets of $X$.

Lemma 2.11. For $T_{1}$-space $X, e(X)=e(A(X))$. 
Theorem 2.12. If $X$ is a star-K-Menger space with $e(X)<\omega_{1}$, then $A(X)$ is star-KMenger.

Proof. We show that $A(X)$ is star-K-Menger. To this end, let $\left(\mathcal{U}_{n}: n \in \mathbb{N}\right)$ be a sequence of open covers of $A(X)$. For each $n \in \mathbb{N}$ and each $x \in X$, choose an open neighborhood $W_{n_{x}}=\left(V_{n_{x}} \times\{0,1\}\right) \backslash\{\langle x, 1\rangle\}$ of $\langle x, 0\rangle$ satisfying that there exists some $U \in \mathcal{U}_{n}$ such that $W_{n_{x}} \subseteq U$, where $V_{n_{x}}$ is an open subset of $X$ containing $x$. For each $n \in \mathbb{N}$, let $\mathcal{V}_{n}=\left\{V_{n_{x}}: x \in X\right\}$, then $\mathcal{V}_{n}$ is an open cover of $X$, thus $\left(\mathcal{V}_{n}: n \in \mathbb{N}\right)$ is a sequence of open covers of $X$, there exists a sequence $\left(K_{n}: n \in \mathbb{N}\right)$ of compact subsets of $X$ such that $\bigcup_{n \in \mathbb{N}}\left\{S t\left(K_{n}, \mathcal{V}_{n}\right): V \in \mathcal{V}_{n}^{\prime}\right\}$ is an open cover of $X$, since $X$ is star-K-Menger. For each $n \in \mathbb{N}$, let $K_{n}^{\prime}=A\left(K_{n}\right)$. Then $K_{n}^{\prime}$ is a compact subset of $A(X)$. Thus we get a sequence $\left(K_{n}^{\prime}: n \in \mathbb{N}\right)$ of compact subsets of $A(X)$ and

$$
X \times\{0\} \subseteq \bigcup_{n \in \mathbb{N}} S t\left(K_{n}^{\prime}, \mathcal{U}_{n}\right)
$$

Let $A=A(X) \backslash \bigcup_{n \in \mathbb{N}} S t\left(K_{n}^{\prime}, \mathcal{U}_{n}\right)$. Then $A$ is a discrete closed subset of $A(X)$. By Lemma 2.11, the set $A$ is countable, we can enumerate $A$ as $\left\{a_{n}: n \in \mathbb{N}\right\}$. For each $n \in \mathbb{N}$, let $K_{n}^{\prime \prime}=K_{n}^{\prime} \cup\left\{a_{n}\right\}$. Then $K_{n}^{\prime \prime}$ is a compact subset of $A(X)$ and

$$
A(X)=\bigcup_{n \in \mathbb{N}} S t\left(K_{n}^{\prime \prime}, \mathcal{U}_{n}\right)
$$

which shows that $A(X)$ is star-K-Menger.

From the proof of Example 2.10, it is not difficult to show the following result.

Theorem 2.13. If $X$ is a $T_{1}$-space and $A(X)$ is a star-K-Menger space. Then $e(X)<\omega_{1}$.

Proof. Suppose that $e(X) \geq \omega_{1}$. Then there exists a discrete closed subset $B$ of $X$ such that $|B| \geq \omega_{1}$. Hence $B \times\{1\}$ is an open and closed subset of $A(X)$ and every point of $B \times\{1\}$ is an isolated point. Thus $A(X)$ is not star-K-Menger by Theorem 2.8, since $B \times\{1\}$ is not star-K-Menger.

We have the following corollary from Theorems 2.12 and 2.13.

Corollary 2.14. If $X$ is a star-K-Menger $T_{1}$-space, then $A(X)$ is star-K-Menger if and only if $e(X)<\omega_{1}$.

\subsection{Acknowledgments}

The author would like to thank Prof. Rui Li for his kind help and valuable suggestions. He would also like to thank the referee for his/her careful reading of the paper and a number of valuable suggestions which led to improvements on several places. 


\section{Bibliography}

[1] M. Bonanzinga, M.V. Matveev, Some covering properties for $\Psi$-spaces, Mat.Vesnik. 61 (2009), 3-11;

[2] J.-L. Cao, Y.-K. Song, Aquaro number versus absolute star-Lindelöf number, Houston J. Math. 29(4) (2003), 925-936;

[3] E. van Douwen, The integers and topology, Handbook of Set-theoretic Topology, North-Holland (K. Kunen and J. E. Vaughan, eds.), Amsterdam (1984), 111-167;

[4] E.K. van Douwen, G.K. Reed, A.W. Roscoe, I.J. Tree, Star covering properties, Topology Appl. 39 (1991), 71-103;

[5] E. Engelking, General Topology, Revised and completed edition, Heldermann Verlag, Berlin, 1989;

[6] W. Just, A.W. Miller, M. Scheepers, P.J. Szeptycki, Combinatorics of open covers(II), Topology Appl. 73 (1996), 241-266;

[7] Lj.D.R. Kočinac, Star-Menger and related spaces, Publ. Math. Debrecen 55 (1999), 421-431;

[8] Lj.D.R. Kočinac, Star-Menger and related spaces II, Filomat (Niš), 13 (1999), 129-140;

[9] M.V. Matveev, A survey on star-covering properties, Topology Atlas, preprint No 330, 1998;

[10] M.V. Matveev, On the extent of SSM spaces, preprint, July (1998);

[11] M. Scheepers, Combinatorics of open covers I: Ramsey theory, Topology Appl. 69 (1996), 31-62;

[12] Y.-K. Song, On star-K-Menger spaces, Hacettepe Journal of Mathematics and Statistics 43(5) (2014), 769-776;

Institute of Mathematics, School of Mathematical Science,

Nanjing Normal University,

Nanjing 210023, P.R. China

email:songyankui@njnu.edu.cn 